\title{
Concise guidance: diagnosis and management of giant cell arteritis
}

\author{
Bhaskar Dasgupta on behalf of the Giant Cell Arteritis Guideline Development Group*
}

\begin{abstract}
Giant cell arteritis (GCA) or temporal arteritis (TA) with polymyalgia rheumatica (PMR) is among the most common reasons for long-term steroid prescription. GCA is a critically ischaemic disease, the most common form of vasculitis and should be treated as a medical emergency. Visual loss occurs in up to a fifth of patients, which may be preventable by prompt recognition and treatment. The British Society for Rheumatology (BSR) and the British Health Professionals in Rheumatology (BHPR) have recently published guidelines on the management of PMR. The purpose of this concise guidance is to draw attention to the full guidelines to encourage the prompt diagnosis and urgent management of GCA, with emphasis on the prevention of visual loss. They provide a framework for disease assessment, immediate treatment and referral to specialist care for management and monitoring of disease activity, complications and relapse.
\end{abstract}

KEY WORDS: diagnosis, giant cell arteritis, steroid therapy, treatment

\section{Introduction}

Giant cell arteritis (GCA) is the most common of all the vasculitides. Together with polymyalgia rheumatic, it represents one of the most common indications for long-term glucocorticosteroid therapy in the community. It is characterised by critical ischaemia at presentation and ranks among the common causes of acute blindness. Visual loss occurs in up to one-fifth of patients and this may relate to late recognition. Early recognition, referral and treatment are essential and GCA should be regarded as a medical emergency. However, it is subject to wide variations in clinical practice, as it is often managed in primary or in secondary care by general practitioners, rheumatologists, non-rheumatologists and ophthalmologists.

Bhaskar Dasgupta, consultant in rheumatology and honorary professor, Essex University Southend University Hospital, Westcliffon-Sea, Essex;

Series editors: Lynne Turner-Stokes and Bernard Higgens; Guest editor: Tabitha Turner-Stokes

*For membership of the guideline development group please see the full guideline ${ }^{1}$

\section{Aims of the guideline}

The objective of this concise guidance is to produce a summary of the longer guidelines, ${ }^{1}$ and to improve the consistency of clinical practice by providing recommendations in the following areas:

- a prompt diagnostic, management and referral process for GCA

- appropriate advice on management of GCA with emphasis on prevention of vision loss with early recognition and prompt high-dose glucocorticosteroid therapy

- investigations (such as temporal artery biopsy) for disease diagnosis and assessment of severity, using clinical factors and histology

- appropriate advice on disease monitoring and treatment of relapses

- appropriate advice on bone protection and prevention of steroid side effects.

Information in this concise guidance has been extracted from the full guideline. Please refer to the full guidance for details of the methodology. The guidelines were developed using the AGREE format and Scottish Intercollegiate Guidelines Network guidance was used to grade the recommendations.

\section{Early diagnosis}

Early recognition and diagnosis of GCA is paramount. Common symptoms and signs are shown in Table 1, and criteria for diagnosis are shown in Box $1 .^{2}$ Clinicians should remember that jaw and tongue claudication, visual symptoms, constitutional symptoms and acute phase response may go unrecognised especially if not accompanied by headaches. ${ }^{3}$ Patients at highest risk of neuro-ophthalmic complications do not always mount high inflammatory responses.

Temporal artery biopsy (TAB) is the cornerstone of diagnosis and often remains positive for two to six weeks after the commencement of treatment. A regular link with a local dedicated surgical unit experienced in TAB to support urgent referrals is recommended. However, TAB may be negative in some patients with GCA, due to the presence of skip lesions or to sub-optimal biopsy. Therefore, patients with negative biopsies should be managed as having GCA if there is a typical clinical and laboratory picture and response to steroids, typical findings on ultrasound, or neuro-ophthalmic features typical of GCA (eg anterior ischaemic optic neuritis). 
Table 1. Common symptoms and signs of giant cell arteritis (GCA)

\begin{tabular}{|c|c|c|}
\hline Symptoms & Signs & Differential diagnoses \\
\hline $\begin{array}{l}\text { - Abrupt onset headache: } \\
\text { - usually unilateral and temporal } \\
\text { - occasionally diffuse or bilateral. } \\
\text { - Scalp pain: } \\
\text { - diffuse or localised } \\
\text { - may lead to difficulty in combing hair } \\
\text { - Jaw and tongue claudication } \\
\text { - Visual symptoms: } \\
\text { - amaurosis fugax, blurring and diplopia } \\
\text { - Constitutional symptoms: } \\
\text { - fever, weight loss and tiredness } \\
\text { - Polymyalgic symptoms } \\
\text { - Limb claudication }\end{array}$ & $\begin{array}{l}\text { - Abnormal superficial temporal artery: } \\
\text { - tender, thickened or beaded } \\
\text { - with reduced or absent pulsation. } \\
\text { - Scalp tenderness } \\
\text { - Transient or permanent visual loss } \\
\text { - Visual field defect } \\
\text { - Relative afferent papillary defect } \\
\text { - Anterior ischaemic optic neuritis: } \\
\text { - pale, swollen optic disc with } \\
\text { haemorrhages on fundoscopy. } \\
\text { - Central retinal artery occlusion: } \\
\text { - unilateral or bilateral. } \\
\text { - Upper cranial nerve palsies } \\
\text { - Features of large vessel GCA: } \\
\text { - asymmetry of pulses and blood } \\
\text { pressure } \\
\text { - bruits (usually of the upper limb). }\end{array}$ & $\begin{array}{l}\text { - Herpes zoster } \\
\text { - Migraine or other causes of headaches } \\
\text { - Serious intracranial pathology eg infiltrative } \\
\text { retro-orbital or base of skull lesions } \\
\text { - Other causes of acute vision loss eg transient } \\
\text { ischaemic attack } \\
\text { - Cervical spine disease } \\
\text { - ENT pathology eg sinus, TMJ and ear disease } \\
\text { - Systemic vasculitides } \\
\text { - Connective tissue diseases }\end{array}$ \\
\hline
\end{tabular}

$\mathrm{TMJ}=$ temporo-mandibular joint; ENT = ear nose and throat.

Box 1. The American College of Rheumatology classification criteria for giant cell arteritis. ${ }^{2}$

For purposes of classification, a patient shall be said to have giant cell (temporal) arteritis if at least three of these five criteria are present.

1 Age at disease onset $\geqslant 50$ years

2 New headache

3 Temporal artery abnormality

4 Elevated erythrocyte sedimentation rate $>50 \mathrm{~mm} / \mathrm{hr}$

5 Abnormal artery biopsy: biopsy specimen showing vasculitis characterised by a predominance of mononuclear cell infiltration or granulomatous inflammation, usually with multinucleated giant cells

Duplex ultrasonography can detect the characteristic appearance of a hypoechoic 'halo', occlusions and stenosis, but requires a high level of experience and training. Positron emission tomography scanning can pick up aortic and large vessel disease; and 3-tesla magnetic resonance imaging has shown mural enhancement in inflamed temporal and occipital arteries. These techniques show promise for the diagnosis and monitoring of GCA (particularly large vessel disease), but currently do not replace TAB for cranial GCA.

\section{Treatment and complications of GCA}

Prompt treatment with high-dose steroids is critical to reduce the likelihood of neuro-ophthalmic complications. The early complications are ischaemic deficits, such as vision loss and stroke. If one eye is affected there is high risk (20-50\%) of bilateral vision loss with further delay or stoppage of treatment. Late complications include the development of aortic aneurysms.

The symptoms of GCA should respond rapidly to highdose glucocorticosteroid treatment, followed by resolution of the inflammatory response. Failure to do so should raise the question of an alternative diagnosis. Steroid-related complications (eg weight gain, fractures, diabetes, hypertension, cataracts and bruising) are also common hence the importance of monitoring and titrating the dose down as soon as it is safe to do so. Low-dose aspirin has also been shown to decrease the rate of visual loss and cerebrovascular accidents in GCA. ${ }^{4}$ In recurrent or resistant GCA, methotrexate or other immunosuppressives (eg azathioprine or leflunomide) may be used as adjuvant therapy to allow reduction in the cumulative glucocorticosteroid dose, or a higher probability of glucocorticosteroid discontinuation without relapse. ${ }^{5}$

\section{Implications and implementation}

There are no cost implications for implementation of these guidelines apart from relevant training for general practitioners who should also have access to the routine investigations outlined and the ability to follow up as suggested. A referral pathway for GCA compatible with these guidelines is suggested in the Map of Medicine.

Further information on the guidelines, ie references, guideline development process, implications and implementation, is 


\section{The guidelines}

\section{Recommendations}

Grade

1 Early recognition and diagnosis

- Clinicians should consider giant cell arteritis (GCA) in patients >50 years presenting with the symptoms listed in Table 1 .

- Particular attention should be paid to the predictive features of neuro-opthalmic complications which are:

- jaw claudication

- diplopia

- temporal artery abnormalities.

\section{Confirmation of diagnosis - temporal artery biopsy}

- Urgent referral for specialist opinion and temporal artery biopsy (TAB) should be considered whenever a diagnosis of GCA is suspected.

However, this should not delay the prompt institution of high-dose glucocorticosteroid therapy.

- Biopsy specimens should be no less than $1 \mathrm{~cm}$, ideally greater than $2 \mathrm{~cm}$, in length. Contra-lateral biopsy is usually not required.

- Patients with negative TAB but a typical clinical picture and response to steroids should be regarded as having GCA.

- Imaging techniques do not replace TAB for diagnosis of cranial GCA.

\section{Large vessel GCA}

- Large vessel GCA should be suspected in patients with prominent systemic symptoms, limb claudication or persistently high-inflammatory markers despite adequate glucocorticosteroid therapy.

- Imaging techniques, such as positron emission tomography and magnetic resonance imaging scanning, should be reserved for the assessment of suspected large vessel involvement

\section{Initial corticosteroid therapy}

- High-dose glucocorticosteroid therapy should be initiated immediately when clinical suspicion of GCA is raised.

- Recommended starting dose regimens are shown in Table 2.

- To minimise the complications of steroid treatment:

- patients should also receive bone protection

- gastrointestinal protection should be considered.

\section{Glucocorticosteroid reduction}

- Steroid reduction should be considered only in the absence of clinical symptoms, signs and laboratory abnormalities suggestive of active disease.

- This should be balanced against the need to use the lowest effective dose, patient wishes and side-effects.

- Recommended dose reduction regimens are shown in Table 2.

\section{Use of low-dose aspirin}

- Should be considered in all patients with GCA, if no contra-indications exist.

\section{Monitoring of therapy}

- Patients should be monitored for evidence of relapse, disease-related and steroid-related complications.

- Monitoring should be clinical and supported by measurement of inflammatory markers. In particular the following features should be sought:

- headaches

- jaw and tongue claudication

- visual symptoms

- vascular claudication of limbs, bruits and asymmetrical pulses

- polymyalgic symptoms

- osteoporotic risk factors and fractures

- other glucocorticosteroid-related complications

- other symptoms that may suggest an alternative diagnosis.

- Recommendations for frequency of follow-up and investigation are shown in Table 2. 


\section{The guidelines - continued}

\section{Relapse}

- Disease relapse should be suspected in patients with a return of symptoms of GCA, ischaemic complications, unexplained constitutional or polymyalgic symptoms. (Relapse is usually associated with a rise in erythrocyte sedimentation rate/C-reactive protein, but may occur with normal inflammatory markers.)

- All patients in whom relapse is suspected should be treated as shown in Table 2, and discussed or referred for specialist assessment.

\section{Recurrent relapse}

- Recurrent relapse or failure to wean glucocorticosteroid dose requires the consideration of adjuvant therapy, such as methotrexate or other immunosuppressants.

- These immunosuppressive agents should be started at the third relapse.

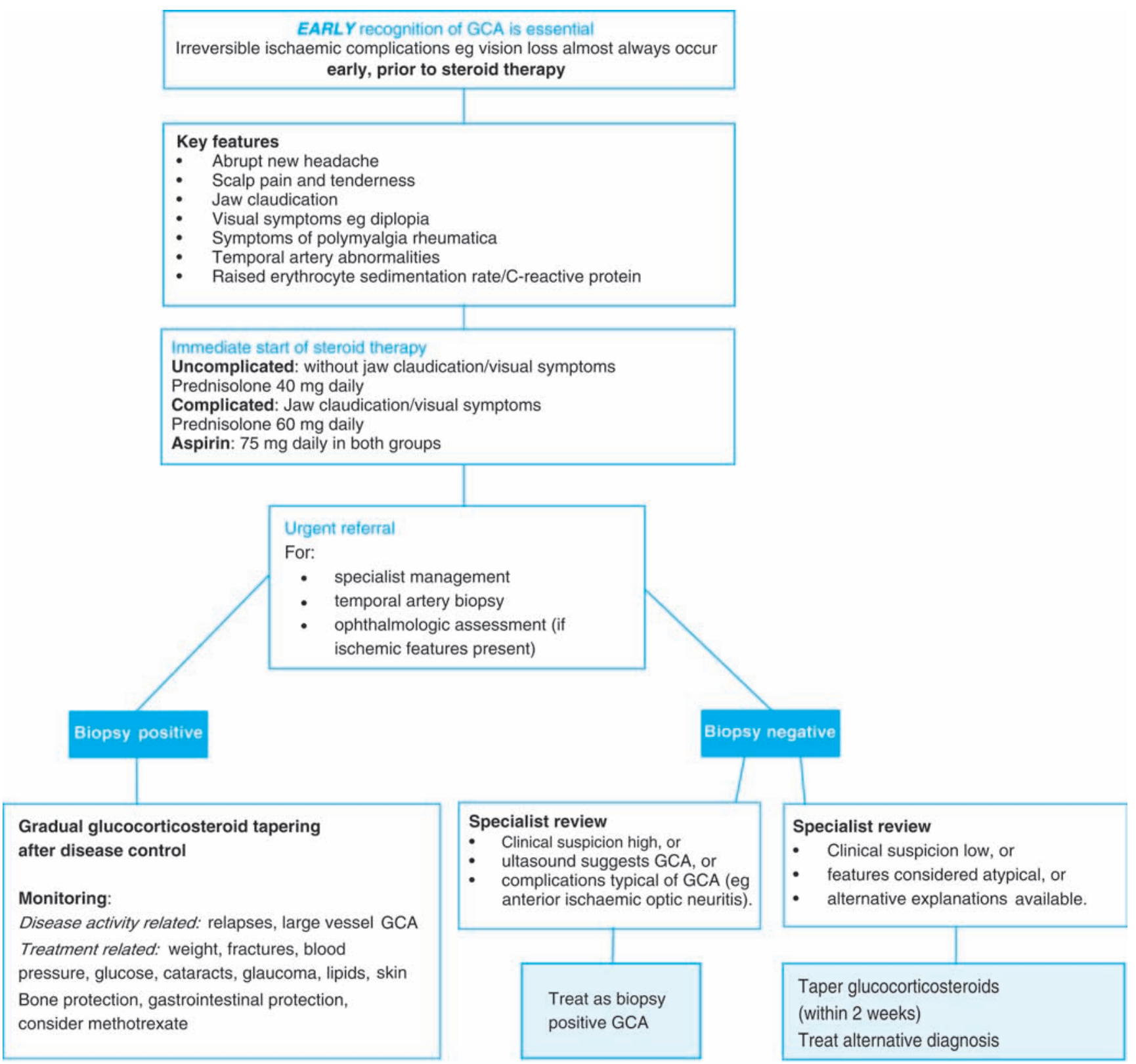

Fig 1. Pathway for management of giant cell arteritis (GCA). 
Table 2. Recommended regimens for glucocorticosteroid treatment.

\section{Starting dose regimens}

Uncomplicated GCA

Complicated GCA

Bone protection

Gastrointestinal protection
No jaw/tongue claudication or visual symptoms:

- prednisolone 40-60 mg (not less than $0.75 \mathrm{mg} / \mathrm{kg}$ ) daily until resolution of symptoms and laboratory abnormalities.

Evolving visual loss or history of amaurosis fugax:

- intravenous methylprednisolone $500 \mathrm{mg}$ to $1 \mathrm{~g}$ daily for three days before oral prednisolone.

Established vision loss:

- at least $60 \mathrm{mg}$ oral prednisolone daily.

Weekly bisphosphonate and calcium/vitamin D supplementation

Protein pump inhibitors (eg omeprazole, lanzoprazole)

The dose may need adjustment, due to disease severity, co-morbid factors (eg diabetes, cardiorespiratory or renal disease), fracture risk, patient wishes, and adverse events. Some patients will require long-term low-dose glucocorticosteroid therapy.

\section{Dose reduction}

Standard regimen

For enteric-coated prednisolone
40-60 mg prednisolone (not less than $0.75 \mathrm{mg} / \mathrm{kg}$ ) continued for four weeks (or until resolution of symptoms and lab abnormalities):

- then dose is reduced by $10 \mathrm{mg}$ every two weeks to $20 \mathrm{mg}$

- then by $2.5 \mathrm{mg}$ every two to four weeks to $10 \mathrm{mg}$

- then by $1 \mathrm{mg}$ every one to two months, provided there is no relapse.

As above down to $10 \mathrm{mg}$ per day. For doses below this:

- $10 \mathrm{mg} / 7.5 \mathrm{mg}$ alternating for two months

- then $7.5 \mathrm{mg}$ daily for one to two months

- then $7.5 / 5 \mathrm{mg}$ alternating for one to two months

- then $5 \mathrm{mg}$ daily for one to two months etc.

Some patients may benefit from a more gradual glucocorticosteroid taper, or a period of treatment at a stable dose, such as $5 \mathrm{mg}$ prednisolone for three months.

\section{Monitoring of treatment}

Frequency of follow-up

Weeks $0,1,3,6$ then months $3,6,9,12$ in the first year

Extra unscheduled visits may be necessary in the event of relapse or adverse events.

\section{Investigations}

At each visit

Every two years
Full blood count, ESR/CRP, urea and electrolytes, glucose

Chest radiograph to monitor for aortic aneurysm (echocardiography, PET and MRI may also be appropriate)

Bone mineral density

\section{Treatment of relapse}

Headache only

Treat with the previous higher glucocorticosteroid dosage

Headache and jaw claudication

Eye symptoms

Large vessel GCA
Treat with 40-60 mg daily prednisolone

Treat with either $60 \mathrm{mg}$ prednisolone or intravenous methylprednisolone

Investigate with imaging techniques (PET, MRI scanning)

Consider treatment using systemic vasculitis protocols

CRP = C-reactive protein; ESR = erythrocyte sedimentation rate; GCA = giant cell arteritis; MRI = magnetic resonance imaging;

PET = positron emission tomography. 
available at Rheumatology online with the full guidelines. ${ }^{1,6}$ An Arthritis Research Campaign booklet on GCA incorporating the guidelines will be published shortly. The booklet has being developed with recommendations from various patient representatives and members of patient organisations such as the Polymyalgia Rheumatica and Giant Cell Arteritis UK.

\section{References}

1 Dasgupta B, Borg FA, Hassan N et al. BSR and BHPR guidelines for the management of giant cell arteritis. Rheumatology (Oxford) 2010 Apr 5 (epub ahead of print).

2 Hunder GG, Bloch DA, Michel BA et al. The American College of Rheumatology 1990 criteria for the classification of giant cell arteritis. Arthritis Rheum 1990;33:1122-8.
3 Borg FA, Salter VLJ, Dasgupta B. Neuro-ophthalmic complications in giant cell arteritis. Curr Allergy Asthma Rep 2008;8:323.

4 Nesher G, Berkun Y, Mates M et al. Low-dose aspirin and prevention of cranial ischemic complications in giant cell arteritis. Arthritis Rheum 2004;50:1332-7.

5 Mahr AD, Jover JA, Spiera RF et al. Adjunctive methotrexate for treatment of giant cell arteritis: an individual patient data meta-analysis. Arthritis Rheum 2007;56:2789-97.

6 Royal College of Physicians. Glucocorticoid-induced osteoporosis: guidelines for prevention and treatment. London: RCP, 2002.

Address for correspondence: Professor B Dasgupta, Department of Rheumatology, Southend University Hospital, Prittlewell Chase, Westcliff-on-Sea, Essex SSO ORY.

Email: bhaskar.dasgupta@southend.nhs.uk.

\section{RCP GUIDELINES}

\section{National clinical guideline for stroke}

\section{Third edition}

\section{Prepared by the Royal College of Physicians Intercollegiate Stroke Working Party co-chaired by Professor Derick Wade and Dr Tony Rudd}

The third edition of these world-renowned stroke guidelines provides the reader with the most comprehensive coverage of stroke care to date, encompassing the whole of the stroke pathway from acute care through to longer-term rehabilitation and secondary prevention. It informs health professionals about what should be delivered to stroke patients and how this should be organised, with the aim of improving the quality of care for everyone who has a stroke, regardless of age, gender, type of stroke, or location.

The recommendations have been completely revised to include the most up-to-date evidence published since the last edition in 2004.

\section{New features included in this guideline}

- Recommendations from the new guideline by the National Institute for Health and Clinical Excellence on the initial management of acute stroke and transient ischaemic attack.

- A new guide for commissioners of stroke services to help ensure that a population receives an integrated high-quality service.

- An updated information booklet for stroke patients and their carers.

- A section on mental capacity and how it influences stroke management.

- Updated sections, on acute care, rehabilitation, longer-term care, and secondary prevention.

- Profession-specific concise guides for nurses, dietitians and therapy professionals.

- A driving section (in relation to UK driving law).

- A laminated concise guide for convenience.

The guidelines are written in a clear and holistic way. They are an essential resource for everyone involved in stroke care, prevention and rehabilitation, as well as commissioners of stroke services, patients and carers.
CLINICAL

EFFECTIVENESS \&

EVALUATION UNIT

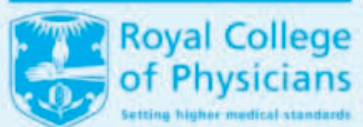

National clinical guideline

for stroke Third edition

Published July 2008

ISBN 978-1-86016-334-0

Price: $£ 36.00$ UK, $£ 46.00$ overseas

Also ovailable

Care after stroke or transient ischaemic attack

Information for patients and their carers (pack of 20 booklets)

ISBN 978-1-86016-342-5

Price: $£ 14.00$ UK, $£ 16.00$ overseas

All prices include postage and packing $10 \%$ discount on all RCP publications to fellows and members

Please quote the reference Clinical Medicine when making your order 